\title{
Conserver un aliment vivant
}

Entretien et circulation d'un ferment : le cas du kéfir

Renaud Debailly, Christophe Lavelle et Émilien Schultz

\section{(2) OpenEdition}

Journals

Édition électronique

URL : https://journals.openedition.org/tc/8971

DOI : $10.4000 /$ tc. 8971

ISSN : 1952-420X

Éditeur

Éditions de l'EHESS

\section{Édition imprimée}

Date de publication : 17 mai 2018

Pagination : 180-183

ISBN : 978-2-7132-2750-9

ISSN : 0248-6016

Référence électronique

Renaud Debailly, Christophe Lavelle et Émilien Schultz, "Conserver un aliment vivant », Techniques \& Culture [En ligne], 69 | 2018, mis en ligne le 17 mai 2020, consulté le 29 septembre 2022. URL : http:// journals.openedition.org/tc/8971; DOI : https://doi.org/10.4000/tc.8971 


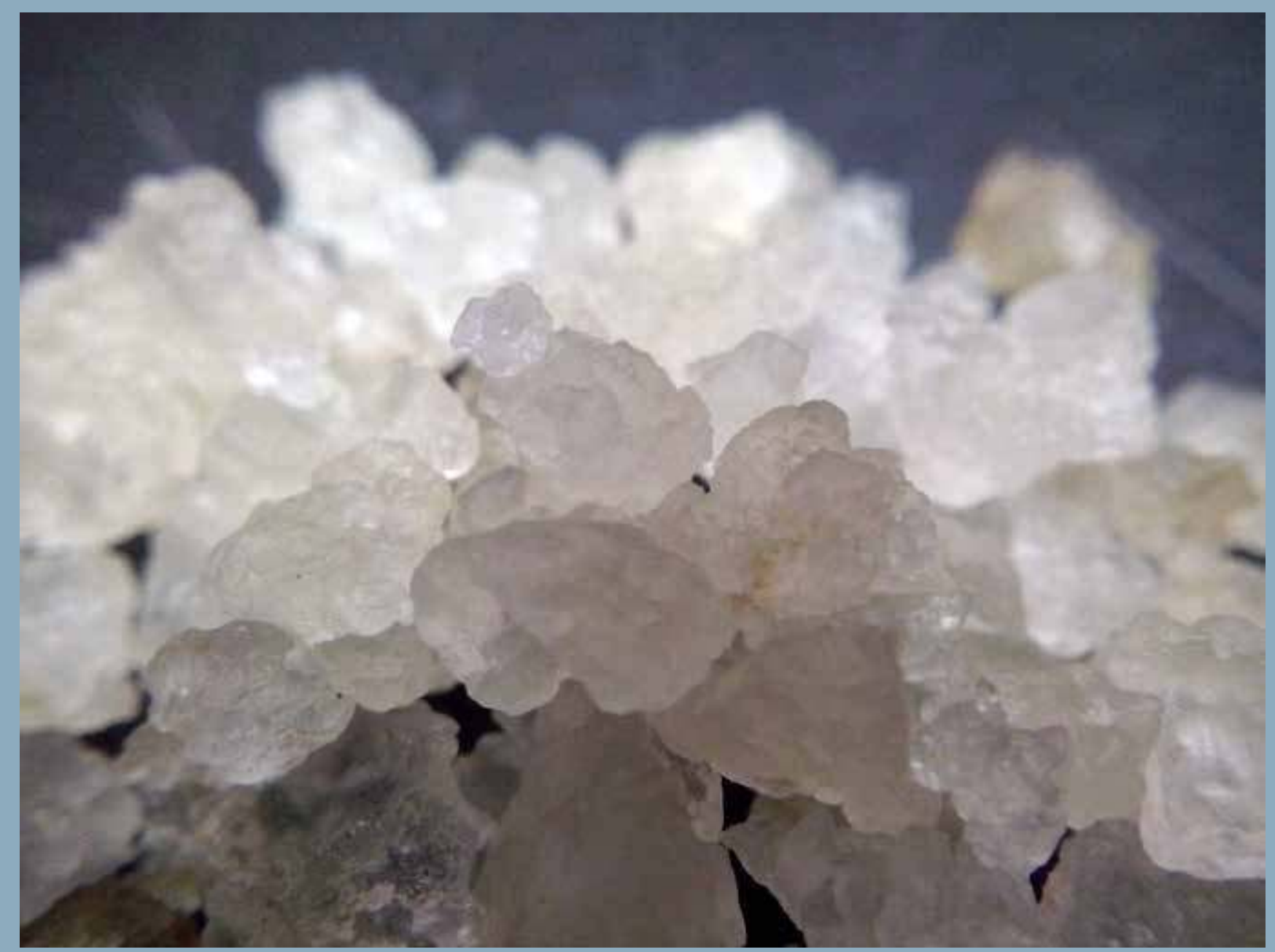




\section{Conserver un aliment vivant Entretien et circulation d'un ferment : le cas du kéfir}

Considérée comme une des plus anciennes méthodes de transformation biochimique des aliments dans l'histoire de l'homme, la fermentation est à l'origine de nombreuses préparations alimentaires, solides comme liquides. Déclenchée spontanément ou favorisée par l'ajout d'un catalyseur biologique (bactérie, levure, champignon), elle est utilisée traditionnellement comme moyen de production, conservation et/ou optimisation nutritionnelle des aliments. Hormis le vin ou la bière issus la plupart du temps d'une fabrication professionnelle, les boissons fermentées «maison» (boza, ginger beer, kéfir, kombucha, pulque, etc.), répandues partout dans le monde, sont dans l'ensemble encore décrites de manière assez incomplète quant à leurs usages et leurs propriétés chimiques, biologiques et physiologiques (Baschali et al. 2017). Le «kéfir» désigne un breuvage pétillant obtenu en utilisant des grains translucides composés d'une symbiose de micro-organismes (Gulitz et al. 2011, Fiorda et al. 2017) dont les propriétés biochimiques ont des conséquences sur les modes de production et de consommation de la boisson.

Comment s'organisent la conservation et la diffusion d'un aliment vivant? Dans le cadre d'une collaboration interdisciplinaire sur le kéfir, nous insistons sur deux spécificités : l'impossibilité de créer ex nihilo les grains confère à leur circulation une importance fondamentale; la conservation et l'évolution de la boisson reposent sur la nécessité de maintenir vivant le ferment pour pouvoir continuer à produire du kéfir.

Comment les incertitudes liées aux transformations de l'aliment vivant sont-elles maîtrisées pendant la transmission des grains comme lors de la production de la boisson? Le kéfir «minimal » commercialisé en pharmacie est très éloigné de par sa composition du kéfir «sauvage», qui circule entre consommateurs. Pour ce dernier, l'impossibilité de créer les grains et de maîtriser leur démultiplication pendant le processus de fermentation implique une circulation du kéfir au sein d'un réseau d'échange en dehors des circuits commerciaux. Plutôt que de jeter l'excédent de grains, les personnes préfèrent souvent les faire circuler dans leur entourage ou 


\section{Les formes de kéfir}

A gauche, le kéfir commercial, produit industriellement et vendu en pharmacie, contenant trois types de bactéries (poudre blanche) et deux types de levures (granulé jaune).

A droite, le kéfir «sauvage », cultivé traditionnellement, renfermant plusieurs dizaines de bactéries et levures différentes dans un substrat gélifié. dans des réseaux impersonnels sur les sites Internet. Préparer cette boisson, c'est donc aussi devenir soi-même un maillon d'une chaîne d'échange qui permet la circulation du ferment et qui repose sur la confiance entre les donneurs et les receveurs. Cette circulation va de pair avec la mise en œuvre de techniques particulières puisque, pour que les grains puissent être stockés, ils doivent être maintenus au frais dans une eau sucrée régulièrement renouvelée, ou, pour une conservation plus longue, déshydratés ou congelés. Ils devront ensuite être réactivés pour ensuite pouvoir réaliser la boisson.

La réalisation de cette boisson fermentée ne repose pas seulement sur l'application d'une recette, elle vise aussi à maintenir vivant les grains pour ne pas avoir à s'en procurer à nouveau. Dans ce contexte, le processus de fermentation est l'objet de représentations vitalistes, la symbiose microbienne composant le grain de kéfir étant perçue comme un organisme autonome dont il faut prendre soin, comme on prendrait soin d'un animal domestique. Il s'agit notamment de ne pas intoxiquer le kéfir avec du métal ou de le «nourrir» à partir de produits eux-mêmes «sains» (donc issus de l'agriculture biologique, non raffinés, etc.). Les recettes viennent ainsi justifier la nécessité de certains aliments, leur degré de pureté, et les gestes à proscrire. Si le kéfir

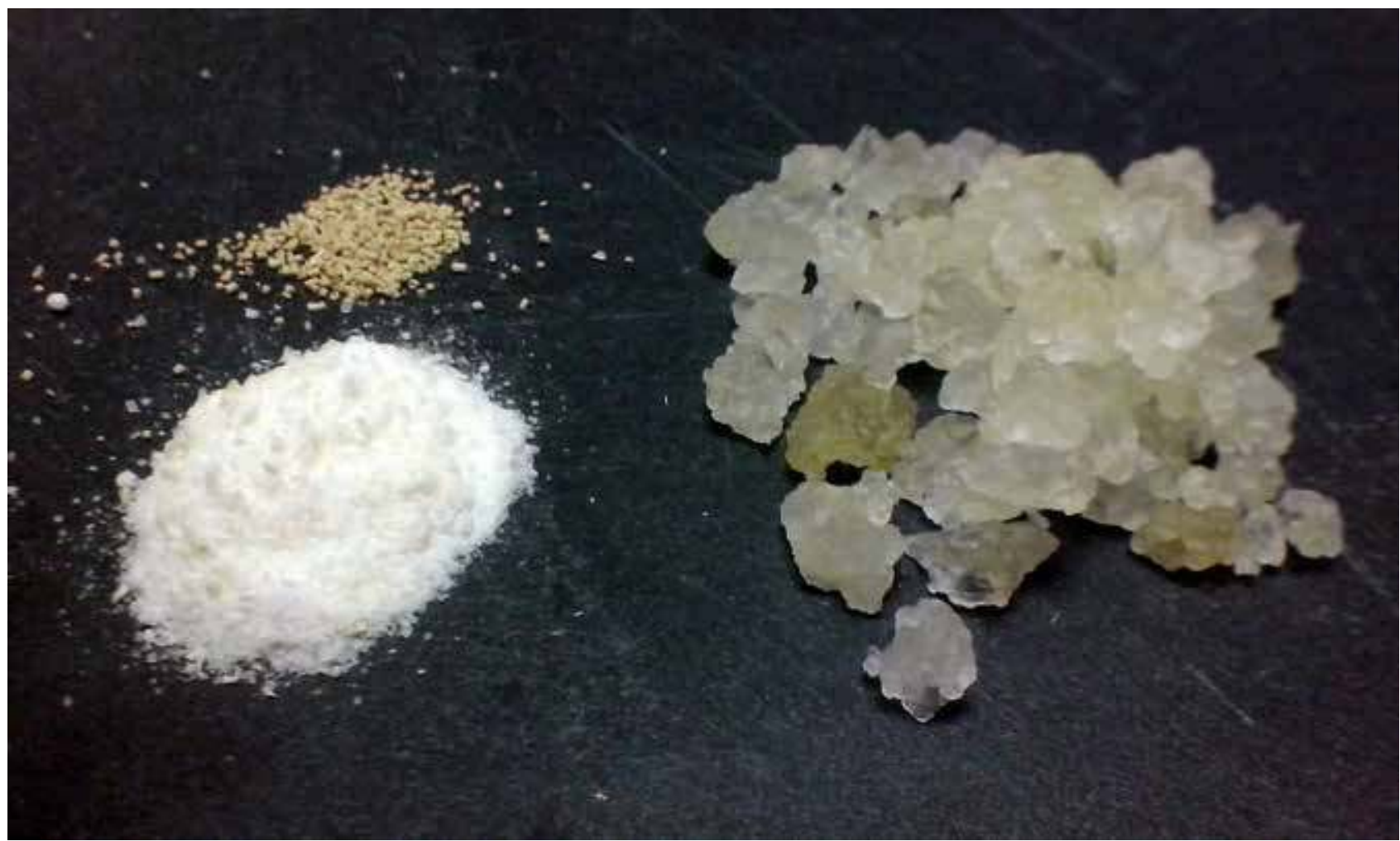


est vu par certains comme une boisson avant tout associée au plaisir de préparer et consommer un aliment en répétant des gestes et des recettes transmis au sein de la sphère familiale, pour d'autres, il est associé à un breuvage ayant des bienfaits pour la santé. L'amélioration de la qualité du produit final occupe alors une place centrale pour en tirer des bénéfices pour soi-même. Ce cas d'étude nous permet de comprendre comment des liens se tissent entre la conservation d'un produit vivant, sa transmission et son entretien au sein de et par des communautés de consommateurs, dans un contexte de bien-être sanitaire visé par le mangeur.

\section{En ligne}

Retrouver l'article complet sur journals.openedition.org/tc: Techniques\&Culture 69 «Le temps des aliments».

\section{Iconographie}

Image d'ouverture. Grains de kéfir. Crédits des images @ Christophe Lavelle.

\section{Les auteurs}

Renaud Debailly est maître de conférences à l'université Paris-Sorbonne et chercheur au sein du Groupe d'étude des méthodes de l'analyse sociologique de la Sorbonne (GEMASS). Ses travaux portent sur les relations sciences-société. Il est l'auteur de La critique de la science depuis 1968 publié chez Hermann en 2015.

Christophe Lavelle est biophysicien, chercheur au CNRS et au Muséum national d'histoire naturelle, à Paris, et co-fondateur du Food 2.0 Lab. Il focalise depuis quelques années ses recherches sur la physico-chimie culinaire et les liens, notamment épigénétiques, entre alimentation et santé. Il est l'auteur de Science culinaire (Belin, 2014).

Émilien Schultz est sociologue, au laboratoire SESSTIM dans l'équipe CanBios et à l'hôpital Gustave Roussy. Ses travaux portent sur la production des savoirs en société. Il mène actuellement des projets sur l'accès des patients aux innovations thérapeutiques en cancérologie.

\section{Références}

Baschali, A., Kyriacou, A., Karavasiloglou, N. \& A. Matalas 2017 «Traditional low-alcoholic and non-alcoholic fermented beverages consumed in European countries: a neglected food group », Nutrition Research Reviews 30: 1-24.

Gulitz, A., Stadie, J., Wenning, M., Ehrmann, M.A. \& R.F. Vogel 2011 «The microbial diversity of water kefir », International Journal of Food Microbiology 151: 284-288. doi: org/10.1016/j.Ijfoodmicro.2011.09.016.

Fiorda, F.A., Melo Pereira de, G.V., Thomaz-Soccol, V., Rakshit, S.K., Pagnoncelli, M.G.B. et al. 2017 «Microbiological, biochemical, and functional aspects of sugary kefir fermentation - A review », Food Microbiol 66: 86-95.

\section{Pour citer l'article}

Debailly, R., Lavelle, C. \& E. Schultz 2018 «Conserver un aliment vivant. Entretien et circulation d'un ferment: le cas du kéfir», TechniquesÉCulture 69 «Le temps des aliments», p. 180-183. 